This item was submitted to Loughborough's Research Repository by the author.

Items in Figshare are protected by copyright, with all rights reserved, unless otherwise indicated.

\title{
The determination of salivary oxypurines before and after exercise by combined liquid chromatography-field asymmetric waveform ion mobility spectrometry-time-of-flight mass spectrometry
}

\section{PLEASE CITE THE PUBLISHED VERSION}

https://doi.org/10.1007/s12127-018-0232-4

\section{PUBLISHER}

(C) Springer

\section{VERSION}

AM (Accepted Manuscript)

\section{PUBLISHER STATEMENT}

This work is made available according to the conditions of the Creative Commons Attribution-NonCommercialNoDerivatives 4.0 International (CC BY-NC-ND 4.0) licence. Full details of this licence are available at: https://creativecommons.org/licenses/by-nc-nd/4.0/

\section{LICENCE}

CC BY-NC-ND 4.0

\section{REPOSITORY RECORD}

Arthur, Kayleigh L., Lynsey S. Wilson, Matthew A. Turner, Martin R. Lindley, James C. Reynolds, and Colin S. Creaser. 2019. "The Determination of Salivary Oxypurines Before and After Exercise by Combined Liquid Chromatography-field Asymmetric Waveform Ion Mobility Spectrometry-time-of-flight Mass Spectrometry". figshare. https://hdl.handle.net/2134/33435. 
The determination of salivary oxypurines before and after exercise by combined liquid chromatography-field asymmetric waveform ion mobility spectrometry-time-of-flight mass spectrometry 


\begin{abstract}
A method combining field asymmetric waveform ion mobility spectrometry with liquid chromatography-mass spectrometry (LC-FAIMS-MS) has been developed for the analysis of the oxypurine compounds hypoxanthine (HX) and xanthine (XA) in saliva. Separation of the oxypurines from interfering matrix components was investigated using FAIMS-MS. The selected FAIMS parameters were then applied to the rapid LC-FAIMS-MS analysis of HX and XA using a short chromatographic separation method (7 min). A comparison of the LC-MS method with and without FAIMS applied, resulted in improved discrimination from saliva matrix interferences and improved chromatographic peak integration for both HX and XA using a FAIMS separation. A quantitative evaluation of the LC-FAIMS-MS method was performed giving limits of detection of $2.0 \mathrm{ng} \mathrm{mL}^{-1}$ for $\mathrm{HX}$ and $1.8 \mathrm{ng} \mathrm{mL}^{-1}$ for XA, and limits of quantification of $6.6 \mathrm{ng} \mathrm{mL}^{-1}$ for $\mathrm{HX}$ and $6.0 \mathrm{ng} \mathrm{mL}^{-1}$ for XA. The developed LC-FAIMS-MS method was applied to the targeted analysis of the oxypurine metabolites in saliva collected from healthy male athletes $(n=11)$ before and after exercise designed to induce oxidative stress; post-exercise collection time-points included immediately after exercise, one hour and twenty-four hours' post-exercise. The salivary concentrations of both HX and XA were lower after physical exercise, compared to the pre-exercise (rest) concentrations and returned to approximately pre-exercise levels after twenty-four hours. The method reported has the potential for monitoring the salivary oxypurines, HX and XA, as biomarkers of oxidative stress and in other clinical applications.
\end{abstract}

\title{
Keywords
}

FAIMS, differential mobility spectrometry, mass spectrometry, oxidative stress, oxypurines, xanthine, hypoxanthine, 


\section{Introduction}

Exhaustive exercise and overtraining can have major health implications for athletes, and exercise induced oxidative stress and inflammation can be associated with many known diseases (Halliwell et al. 1993; Vollaard et al. 2005). The identification of biomarkers of oxidative stress could therefore lead to the early identification and effective treatment of diseases and chronic inflammation (Margonis et al. 2007). Exercise induced muscle fatigue or muscular stress has previously been related to oxidative stress, ATP depletion, muscle damage or immunological compromise (Speranza et al. 2007; Al-Shehri 2015; Li 2015; Finsterer et al. 2016; Chan 2018).

The oxypurine compounds hypoxanthine (HX) and xanthine (XA) are naturally occurring purine derivatives (Figure 1), which are derived from the degradation of purine nucleotides (adenine and guanine). They have been positively correlated with ATP consumption and the formation of free radicals and reactive oxygen species (Finsterer 2012; Radak et al. 2008). HX is also produced by the action of xanthine oxidase on XA, or from the reduction of XA by xanthine oxidoreductase (XOR). In the purine catabolism, XOR catalyses the oxidative hydroxylation of HX to XA and subsequently of XA to uric acid (UA). UA acts as a potent antioxidant and free radical scavenger associated with oxidative stress and other diseases (Guan et al. 2004, 2005; Yigla et al. 2007). XOR therefore has important functions as a cellular defence enzyme against oxidative stress (Valko et al. 2007). HX and $\mathrm{XA}$ are usually analysed in plasma, serum or urine, and it has been reported that plasma and or urinary levels of these oxypurines can changes as a result of oxidative stress (Catinella et al. 2001; Murray 1966). In a study of twenty healthy males performing exercise to exhaustion, serum HX and XA levels were found to significantly increase post exercise, leading to the proposal of oxypurines as biomarkers of oxidative stress and muscle fatigue (Speranza et al. 2007). Similar results were also observed in the analysis of HX and XA in plasma where an increase in concentrations of both HX and XA were observed post-exercise, with a peak increase in the levels of HX post exercise of nearly nine times the resting level (Hellsten-Westing et al. 1994). HX has been suggested as an indicator of the effectiveness of a training regime (Zielinski et al. 2011), and as a potential biomarker to normalise for metabolomic changes due to exercise, although further research is required (Daskalaki et al. 2015, 2014).

HX and XA have been detected in plasma, serum or urine using a variety of methods including high performance liquid chromatography (HPLC) and capillary electrophoresis (Guan et al. 2004, 2005; Markelj et al. 2016). These oxypurines have also been detected in saliva (Al-Shehri et al. 2013; Guan et al. 2004, 2005; Kochanska et al. 2000; Markelj et al. 2016), which is known to change in response to a variety of different physiological states (Dame et al. 2015). However, saliva is a complex biofluid which can require careful sample preparation and/or extended analysis times to separate HX and XA from co-eluting matrix interferences. This results in the relatively long analysis times (15-60 min) 
reported for the liquid chromatography-mass spectrometry (LC-MS) analysis of HX, XA and structurally related purine compounds in saliva and other biofluids (Al-Shehri et al. 2013; Flanders et al. 2005; Ito et al. 2000; Lu et al. 2006; Ong et al. 2010).

The performance of liquid chromatographic separation can be improved by the use of ultra-high performance liquid chromatography (UHPLC) and high resolution mass spectrometry (HRMS), using time-of-flight (TOF) or Orbitrap mass analysers, or by tandem MS. However, even the highest resolution mass analysers cannot separate isobaric analytes and matrix interferences. An alternative approach to enhancing the selectivity and performance of LC-MS analyses is the incorporation of a rapid gas-phase ion separation technique such as ion mobility spectrometry (IMS) in combination with LC-MS using either drift tube IMS or field asymmetric waveform ion mobility spectrometry (FAIMS), also known as differential ion mobility spectrometry. FAIMS separates ions based on differences in the mobility of ions in low and high electric fields, which is a separation mechanism that is highly orthogonal to LC and MS. The combination of a miniaturised FAIMS device with LCMS has been shown to improve selectivity and limits of detection and quantification without loss of linear dynamic range, whilst reducing interfering chemical noise in the analysis of complex biofluids (Arthur et al. 2017; Brown et al. 2012; Smith et al. 2013). Here, we report the development of a rapid LC-FAIMS-MS method, requiring minimal sample pre-treatment, for the determination of the oxypurines HX and XA in saliva. The method has been applied to the analysis of saliva samples collected from male cyclists pre- and post-exhaustive exercise. 


\section{Experimental}

\section{Chemicals}

HPLC grade acetonitrile (ACN) and HPLC grade water $\left(\mathrm{H}_{2} \mathrm{O}\right)$ were both purchased from Fisher Scientific (Loughborough, UK). HX, XA, 1-methylxanthine-(methyl $\left.-{ }^{13} \mathrm{C}, \mathrm{d}_{3}\right)\left(1-\mathrm{MX}_{\mathrm{d} 3}\right)$ and formic acid (FA) were all purchased from Sigma Aldrich (Dorset, UK). Individual standard solutions of HX (1 $\mu \mathrm{g}$ $\left.\mathrm{mL}^{-1}\right)$, XA $\left(1 \mu \mathrm{g} \mathrm{mL}^{-1}\right)$ and 1-MX $\mathrm{d}_{3}\left(0.5 \mu \mathrm{g} \mathrm{mL}^{-1}\right)$ were prepared in $\mathrm{H}_{2} \mathrm{O}: \mathrm{ACN} \mathrm{v} / \mathrm{v}$ 95:5 with $0.1 \% \mathrm{FA}$ for FAIMS-MS analysis. A solution of 1-MX $\mathrm{MX}_{\mathrm{d} 3}\left(0.3 \mu \mathrm{g} \mathrm{mL}^{-1}\right)$ was prepared in $\mathrm{H}_{2} \mathrm{O}$ :ACN v/v 92.5:7.5 with $0.15 \%$ FA for spiking into saliva for LC-FAIMS-MS analysis of saliva samples. Calibration standard solutions of HX and XA were prepared in $\mathrm{H}_{2} \mathrm{O}: \mathrm{ACN}$ v/v 92.5:7.5 with 0.15\% FA for spiking into a pooled saliva extract during sample preparation to give final concentrations (post-spiking) of $\mathrm{HX}$ and XA of 20, 40, 60, 80 and $100 \mathrm{ng} \mathrm{mL}^{-1}$, and $200 \mathrm{ng} \mathrm{mL}^{-1}$ of 1-MX $\mathrm{MX}_{\mathrm{d} 3}$, along with an extract spiked only with 1-MX $\mathrm{d} 3$.

\section{Saliva sample collection}

Saliva samples were collected from trained male cyclists $(n=11)$ aged between $18-45$. Prior to the exercise performance test, participants were asked to arrive in a euhydrated and fasted state (from 10 $\mathrm{pm}$ the day prior to the test) and to refrain from caffeinated and alcoholic beverages for twenty-four hours prior to the test. Ethical approval for the study was obtained from the local ethical advisory committee (Ethical Advisory Committee, Loughborough University) with reference numbers: R14P72, G10-P24 and G10-P23. Saliva was collected from participants pre-exercise, immediately postexercise, one-hour post-exercise and twenty-four hours post exercise. The exhaustive exercise consisted of cycling on a cycle ergometer sub maximally at $70 \%$ of their maximum power output (determined via a pre-testing session to measure maximum power output) for 45 minutes and then to cycle the greatest distance possible for a further 15 minutes. The one hour cycling exercise protocol was designed to induce oxidative stress (Wilson et. al. 2017).

Saliva was collected by a passive drool method where participants were asked to sit comfortably with their head tilted forward, to allow saliva to pool in the front of the mouth (Chiappin et al. 2007; Malkar et al. 2013). Saliva was dribbled from the mouth into a glass collection vial (30 mL, Chromacol, UK) at regular intervals. This process was continued for at least 5-10 minutes to provide $1.5-2 \mathrm{~mL}$ of saliva. Saliva collected from all participants was re-aliquoted into $600 \mu \mathrm{L}$ aliquots into microcentrifuge tubes ( $2 \mathrm{~mL}$ LoBind Eppendorf, Sigma Aldrich, UK). $600 \mu \mathrm{L}$ from all participants was pooled together and re-aliquoted as $600 \mu \mathrm{L}$ aliquots to be used for method development and quality control (QC). Samples were aliquoted over ice prior to storage to minimise freeze-thaw cycles and stored at $-80^{\circ} \mathrm{C}$ to arrest biological activity. 


\section{Saliva sample pre-treatment}

Saliva samples stored at $-80^{\circ} \mathrm{C}$ were thawed at room temperature for $20 \mathrm{~min}$. Each sample was vortexed for $30 \mathrm{~s}$ followed by centrifugation at $10,000 \mathrm{~g}$ for $10 \mathrm{~min}$ at ambient temperature. An aliquot of the supernatant $(250 \mu \mathrm{L})$ was transferred to a fresh microcentrifuge tube and diluted with $500 \mu \mathrm{L}$ of an internal standard solution $\left(1-\mathrm{MX}_{\mathrm{d} 3}\left(0.3 \mu \mathrm{g} \mathrm{mL}^{-1}\right)\right.$ in $\mathrm{H}_{2} \mathrm{O}: \mathrm{ACN} \mathrm{v} / \mathrm{v}$ 92.5:7.5 with $0.15 \%$ FA) to prepare the sample at the starting mobile phase conditions $\left(\mathrm{H}_{2} \mathrm{O}: \mathrm{ACN} \mathrm{v} / \mathrm{v} 95: 5\right.$ with $\left.0.1 \% \mathrm{FA}\right)$, giving a final internal standard concentration of $0.2 \mu \mathrm{g} \mathrm{mL}-1$. The diluted supernatant was vortexed for $30 \mathrm{~s}$ to ensure homogeneity and transferred to an autosampler vial $(2 \mathrm{~mL})$ for LC-FAIMS-MS analysis.

\section{Instrumentation}

Analysis was performed using an Agilent 1200 series HPLC interfaced to an Agilent 6230 TOF mass spectrometer fitted with a modified Jet Stream electrospray ionisation (ESI) source (Agilent Technologies, Santa Clara, USA) to accommodate the FAIMS. The miniaturised chip-based ultraFAIMS device (Owlstone Medical Ltd), described in detail elsewhere (Brown et al. 2010), was located in front of the transfer capillary of the MS behind a modified spray shield within the ESI source. The electrodes of the FAIMS chip are arranged as multiple parallel channels, with a trench length of $78.1 \mathrm{~mm}$, a $100 \mu \mathrm{m}$ electrode gap and a path length of $700 \mu \mathrm{m}$. Nitrogen $\left(\mathrm{N}_{2}\right)$ was used as the carrier gas for the FAIMS and the MS drying gas. Samples were introduced into the ESI source either by direct syringe infusion at a flow rate of $10 \mu \mathrm{L} \mathrm{min}^{-1}$ (for initial FAIMS optimisation by FAIMS-MS) or by the LC. Chromatographic separations were performed on an Agilent Poroshell 120 EC-C18 reversed phase column of dimensions 2.1 x 75 mm, $2.7 \mu \mathrm{m}$ (Agilent Technologies, Santa Clara, USA), with a $5 \mathrm{~mm}$ guard column of the same phase attached in series prior to the analytical column.

\section{HPLC conditions}

Mobile phase A consisted of $0.1 \%$ aqueous formic acid (v/v) and mobile phase B was $0.1 \%$ formic acid in ACN. The mobile phase flow rate was set to $0.3 \mathrm{~mL} \mathrm{~min}^{-1}$ and the column was maintained at $40^{\circ} \mathrm{C}$. Saliva samples were analysed with an injection volume of $10 \mu \mathrm{L}$ by the chromatographic gradient programme: $5 \% \mathrm{~B}(0 \mathrm{~min})$, increased to $8 \% \mathrm{~B}(0-2 \mathrm{~min})$ for the retention of the oxypurine compounds, followed by a column clean-up and equilibration phase by increasing from $8 \%$ B to $95 \%$ B (2-3 min), hold (3-4 min), then returning to initial conditions of 5\% B (4-5 min) and hold (5-7 min).

\section{MS Conditions}

The TOF-MS was operated in positive ion mode with a scan rate of 10 scans s$^{-1}$ for FAIMS-MS analysis, and at 2 scans s$^{-1}$ for LC-FAIMS-MS analysis. For all analyses the MS parameters were as follows: $\mathrm{m} / \mathrm{z}$ range $50-1200$, nebulizer pressure was set to $35 \mathrm{psig}$ with a sheath gas $\left(\mathrm{N}_{2}\right)$ flow of $11 \mathrm{~L}$ 
$\mathrm{min}^{-1}$ at $350^{\circ} \mathrm{C}$, with a nozzle voltage of $2000 \mathrm{~V}$. The capillary voltage was set to $4000 \mathrm{~V}$, the fragmentor voltage was set to $150 \mathrm{~V}$ and the drying gas $\left(\mathrm{N}_{2}\right)$ flow set to $10 \mathrm{~L} \mathrm{~min}^{-1}$ at $150^{\circ} \mathrm{C}$.

\section{Optimisation of FAIMS conditions}

FAIMS-MS analyses of HX and XA were performed using a two-dimensional FAIMS scan (or sweep) in the dispersion field (DF) range from 180-300 Td, in steps of $10 \mathrm{Td}$, and compensation field (CF) range from $-2-5 \mathrm{Td}$, in steps of $0.05 \mathrm{Td}$ (where $1 \mathrm{Td}=10^{-17} \mathrm{~V} \mathrm{~cm}^{2}$ ). The $\mathrm{DF}$ and $\mathrm{CF}$ selected for the LC-FAIMS-MS analysis of saliva were optimised as DF $230 \mathrm{Td}$ and CF $0.90 \mathrm{Td}$ with the TOFMS operated in positive ion mode.

\section{Analytical protocol for LC-FAIMS-MS salivary analysis}

The sample list for analysis was randomised using Microsoft Excel. A series of five consecutive injections of a pooled saliva extract were run prior to sample analysis to condition the chromatographic column. A pooled saliva extract was analysed after every five saliva samples as a QC sample. Method blanks demonstrated no carry-over between saliva sample runs.

\section{Data Processing}

Data were acquired using the FAIMS Control software in combination with the MassHunter Acquisition software. MassHunter Qualitative Analysis software version B.05.00 (Agilent Technologies, Santa Clara, USA) was used for processing raw LC-MS and LC-FAIMS-MS data. A modified prototype version of the MassHunter Qualitative Analysis software was used for processing raw FAIMS-MS data, with a mass extraction window of $m / z \pm 0.02$. Microsoft Excel and PowerPoint 2010 (Microsoft, Seattle, USA), and OriginLab 2015 (Academic version, b9.2.272) were used to produce figures in this report. 


\section{Results and Discussion}

\section{LC-FAIMS-MS method development for the analysis of $H X$ and XA}

A short chromatographic method (7 min) was developed for HX, XA and the internal standard 1$\mathrm{MX}_{\mathrm{d} 3}$ with the analytes eluting in $<2 \mathrm{~min}$ and the remainder of the chromatographic method used to wash-out the saliva matrix and re-equilibrate the column. The retention times for the extracted ion chromatograms (EICs) of the $[\mathrm{M}+\mathrm{H}]^{+}$ions for $\mathrm{HX}\left(\mathrm{m} / z\right.$ 137.0458, $\left.100 \mathrm{ng} \mathrm{mL}^{-1}\right), \mathrm{XA}(\mathrm{m} / z 153.0407$, $100 \mathrm{ng} \mathrm{mL}^{-1}$ ) and 1-MX $\mathrm{d}_{\mathrm{d} 3}\left(\mathrm{~m} / z\right.$ 171.0791, $200 \mathrm{ng} \mathrm{mL}^{-1}$ ) are $0.88 \mathrm{~min}$ (Figure 2 (a)), $0.95 \mathrm{~min}$ (Figure 2 (b)) and $1.62 \mathrm{~min}$ respectively. In the EICs for $\mathrm{m} / \mathrm{z} 137.0458$ and $\mathrm{m} / \mathrm{z} 153.0407$ from an unspiked pooled saliva extract (Figure 2 (c) and (d)), co-eluting matrix interferences are observed, with HX (Figure 2 (c)) unresolved from these interferences and XA is not baseline resolved (Figure 2 (d)). Selection of optimal FAIMS conditions for the targeted analysis of HX and XA in saliva by LCFAIMS-MS was determined by the removal of co-eluting matrix interferences to improve the signalto-noise ratio (S:N), peak area, peak height and peak shape compared to LC-MS alone. The oxypurine compounds and the internal standard (1-MX $\left.\mathrm{MX}_{\mathrm{d} 3}\right)$ were first analysed as individual standard solutions along with an unspiked pooled saliva extract (post sample preparation). The samples were directly infused into the ESI source and analysed by FAIMS-MS, in order to select FAIMS DF and CF parameters to be further tested using LC-FAIMS-MS. FAIMS-MS analysis was performed by scanning the DF from 180 to $300 \mathrm{Td}$ in $10 \mathrm{Td}$ steps and the CF from -2 to $5 \mathrm{Td}$ in $0.05 \mathrm{Td}$ steps. Figure 3 (a) shows that ions from the saliva extract are observed in the CF range from -2 to $3 \mathrm{Td}$ at DF $230 \mathrm{Td}$, with the highest intensity at $2 \mathrm{Td}$. The selected CF responses for HX and XA in Figure 3 (a) are in the $\mathrm{CF}$ range 0 to $1.5 \mathrm{Td}$ overlapping the $\mathrm{CF}$ total ion chromatogram (TIC) which shows the entire ion response obtained from the saliva extract. Three DF/CF conditions were selected where HX and XA are transmitted by the FAIMS along with the internal standard 1-MX $\mathrm{X}_{\mathrm{d} 3}$ : CF $0.7 \mathrm{Td}$ at DF 220 Td (Figure 3 (b)), CF 0.9 Td at DF 230 Td (Figure 3 (c)), and CF 1.1 Td at DF 240 Td (Figure 3 (d)). The three FAIMS conditions selected were applied to the LC-FAIMS-MS analysis of an unspiked saliva extract in order to determine the optimum DF/CF combination for the transmission of HX, XA and 1-MX $\mathrm{M}_{\mathrm{d} 3}$. EICs for $\mathrm{HX}(\mathrm{m} / \mathrm{z} 137.0458 \pm 0.02,0.91 \mathrm{~min})$ and XA $(\mathrm{m} / z 153.0407 \pm 0.02,0.96 \mathrm{~min})$ are shown in Figure 4. Each of the three FAIMS conditions resulted in a reduction of the co-eluting interferences in the EICs for HX (Figure 4 (b) to (d)) and XA (Figure 4 (f) to (h)) compared to LCMS (Figure 4 (a) and (e)). The S:N ratio of both HX and XA was increased in comparison to LC-MS for all three FAIMS conditions by an average of $230 \%$ for HX and $255 \%$ for XA. CF $1.1 \mathrm{Td}$ at DF $240 \mathrm{Td}$ resulted in the largest reduction of co-eluting matrix interferences, but also had the largest reduction in the peak intensity at the highest DF. CF $0.7 \mathrm{Td}$ at DF $220 \mathrm{Td}$ had the highest peak intensity, but the least reduction in the co-eluting matrix interferences. CF $0.9 \mathrm{Td}$ at DF $230 \mathrm{Td}$ was 
therefore selected as the FAIMS condition for the analysis as a compromise between peak intensity and separation of co-eluting matrix interferences.

The LC-MS chromatographic method was developed to minimise the run time for HX and XA, but this resulted in a significant overlap of the HX peak with salivary matrix components. The observed co-elution of HX with matrix components could be reduced by changing the chromatographic gradient to separate the metabolite from the saliva matrix, at the cost of increased chromatographic run time. Options to improve the selectivity of HX without increasing chromatographic run time include the selection of a narrower mass extraction window using HRMS or the application of FAIMS separation combined with LC-MS (Smith et al. 2013). A direct comparison of both techniques to improve selectivity for HX without increasing the run time is shown in Figure 5. The LC-MS and LCFAIMS-MS EICs for the $[\mathrm{HX}+\mathrm{H}]^{+}$ion $(\mathrm{m} / z$ 137.0458) for the analysis of $\mathrm{HX}$ in an unspiked pooled saliva sample, with a high resolution mass window of $m / z 137.0458 \pm 0.02$ is shown in Figure 5 (a). The absolute intensity of the HX peak, with FAIMS switched off, is reduced by a factor of two if the mass extraction window is narrowed from \pm 0.02 (Figure 5 (a)) to $m / z 137.0458 \pm 0.008$ (Figure 5 (b)), but there is no further discrimination against the co-eluting saliva matrix interference. In contrast, the application of FAIMS separation shows a significant reduction in the co-eluting chemical noise from the saliva matrix (Figure 5 (c) and (d)) whilst maintaining the rapid elution time. The removal of chemical noise from the saliva matrix resulted in improved peak integration and S:N even though the absolute intensity of the HX peak was reduced due to ion losses in the FAIMS device. Comparison of the two mass extraction windows with FAIMS on, $m / z 137.0458 \pm 0.02$ (Figure 5 (c)) and $\mathrm{m} / \mathrm{z}$ $137.0458 \pm 0.008$ (Figure $5(\mathrm{~d})$ ), resulted in a reduction of sensitivity without a significant increase in $\mathrm{S}: \mathrm{N}$. Therefore, the \pm 0.02 mass extraction window was selected for subsequent LC-FAIMS-MS analyses. The mass spectrum from the HX peak with the FAIMS off showed HX with a mass error of -9.6 ppm ( $m / z$ 137.0471). Whereas with FAIMS applied (LC-FAIMS-MS) the observed mass error was $-0.82 \mathrm{ppm}(\mathrm{m} / z$ 137.0459), as a result of discrimination against isobaric co-eluting saliva matrix interferences.

Table 1 compares the quantitative performance of the LC-MS and LC-FAIMS-MS methods for the determination of HX and XA using standard additions spiked into a pooled saliva sample. From Table 1, the limit of detection (LOD; S:N 3:1)) for HX was reduced from $3.8 \mathrm{ng} \mathrm{mL}^{-1}$ (FAIMS off) to $2.0 \mathrm{ng}$ $\mathrm{mL}^{-1}$ (FAIMS on), and the limit of quantification (LOQ; S:N 10:1) was also reduced from $12.6 \mathrm{ng}$ $\mathrm{mL}^{-1}$ (FAIMS off) to $6.6 \mathrm{ng} \mathrm{mL}^{-1}$ (FAIMS on), based on the extracted ion peak areas of $\mathrm{HX}(\mathrm{m} / z$ $137.0458 \pm 0.02)$ using LC-FAIMS-MS. The LOD and LOQ for XA were similar at $1.9 \mathrm{ng} \mathrm{mL}^{-1}$ and $6.2 \mathrm{ng} \mathrm{mL}^{-1}$ (FAIMS off) and $1.8 \mathrm{ng} \mathrm{mL}^{-1}$ and $6.0 \mathrm{ng} \mathrm{mL}^{-1}$ (FAIMS on), based on the extracted ion peak areas of XA $(\mathrm{m} / \mathrm{z} 153.0407 \pm 0.02)$ using LC-FAIMS-MS. The intra-day reproducibility of the LC-FAIMS-MS system was compared with LC-MS by analysing HX and XA spiked into saliva (100 ng $\mathrm{mL}^{-1}$, Table 1) in both FAIMS on and FAIMS off modes. Relative standard deviations (\%) 
sufficient for good quantitation were obtained with both LC-MS and LC-FAIMS-MS (1.7\% - 6.6\%). These data for HX demonstrate the FAIMS device can enhance quantitative performance compared to high resolution LC- MS.

\section{LC-FAIMS-MS determination of salivary hypoxanthine and xanthine pre- and post-}

\section{oxidative stress}

The LC-FAIMS-MS method developed for the analysis of HX and XA was applied to the analysis of saliva samples collected from cyclists pre-exercise, immediately post-exercise, one-hour post exercise and twenty-four hours post exercise. A large biological variance between participants was observed in the pre-exercise saliva samples with concentrations in the range $3.1-242.9 \mathrm{ng} \mathrm{mL}^{-1}(0.02-1.8 \mathrm{uM})$ for HX and 22.4 to $1183.1 \mathrm{ng} \mathrm{mL}^{-1}(0.15-7.8 \mathrm{uM}$ ) for XA (Supplementary Table 1 and Supplementary Table 2). These XA and HX concentrations are within the ranges previously reported in saliva, although at the lower end of the range for HX (Kochanska et al. 2000; Al-Shehri 2013; AlShehri 2015). Figure 6 shows box and whisker plots of HX and XA concentrations for male participants pre- and post-exercise. Despite the biological variance in the salivary HX and XA levels pre-exercise, the same overall trend towards lower concentrations immediately after exercise and for the next hour post-exercise is observed, with the levels of HX and XA returning to approximately preexercise levels in the twenty-four hours post-exercise samples. The observation of lower HX and XA levels in saliva after the physical exercise (Figure 6) is the opposite of the trend previously reported for plasma concentrations of $\mathrm{HX}$, which increased immediately post-exhaustive physical exercise (Hellsten-Westing et al. 1994; Speranza et al. 2007). Lower levels of HX and XA (Figure 6) may be due to changes in enzymatic activity in saliva after exercise (Ligtenberg et al. 2015; Walsh 1999). However, significant inter-day variations in salivary levels of HX and XA (and UA) have been reported by Kochańska et al., which may explain the variations in HX and XA concentrations observed in this study. Markelj et al. (Kochanska et al. 2000; Markelj et al. 2016) reported the analysis of saliva and urine by LC-MS, which showed that concentrations of HX and XA were significantly lower, relative to the concentration of UA, in saliva than in urine, although salivary UA has been shown to have a linear correlation with serum UA concentrations (Soukup et al. 2012). Further work is required to understand the relative levels of HX, XA and UA in saliva as a result of oxidative stress. 


\section{Conclusion}

A rapid (7 min) method combining miniaturised FAIMS with LC-MS and non-invasive sampling has been developed for the determination of HX and XA in saliva. Using LC-FAIMS-MS, improved chromatographic peak integration was possible, in comparison to LC-MS, due to the reduction in the co-eluting chemical interferences from the saliva matrix. This also resulted in improved mass spectrometric observed mass error using HRMS, aiding in the qualitative identification and quantitation of the purine based derivatives, HX and XA. Saliva samples analysed from healthy male athletes showed a decrease in salivary oxypurines immediately post-exercise and one-hour postexercise, in comparison to levels determined pre-exercise and twenty-four hours' post-exercise, which suggests that both HX and XA could be used as biomarkers of oxidative stress. The combination of LC-FAIMS-MS with the non-invasive sampling collection technique for saliva has potential as a rapid and high-throughput screening technique for oxidative stress, based on the determination of oxypurine metabolite biomarkers, as well as in other clinical applications.

\section{Acknowledgements}

The authors thank Owlstone Ltd. and Loughborough University for financial support, and Owlstone Ltd. and Agilent Technologies for the provision of instrumentation. 


\section{References}

Al-Shehri S S, Henman M, Charles B G, Cowley D, Shaw P N, Liley H, Tomarchio A, Punyadeera C, \& Duley J A (2013) Collection and determination of nucleotide metabolites in neonatal and adult saliva by high performance liquid chromatography with tandem mass spectrometry. J Chromatogr B 931:140-147

Al-Shehri S, Knox C L, Liley H G, Cowley D M, Wright J R, Henman M G, Hewavitharana A K, Charles B G, Shaw P N, Sweeney E L, Duley J A (2015) Breastmilk-saliva interactions boost innate immunity by regulating the oral microbiome in early infancy. PLoS ONE 10:1-19

Arthur K L, Turner M A, Brailsford A D, Kicman A T, Cowan D A, Reynolds J C, \& Creaser C S (2017) Rapid Analysis of Anabolic Steroid Metabolites in Urine by Combining Field Asymmetric Waveform Ion Mobility Spectrometry with Liquid Chromatography and Mass Spectrometry. Anal Chem 89:7431-7437

Brown L J, Smith R W, Toutoungi D E, Reynolds J C, Bristow A W T, Ray A, Sage A, Wilson I D, Weston D J, Boyle B, \& Creaser C S (2012) Enhanced analyte detection using in-source fragmentation of field asymmetric waveform ion mobility spectrometry-selected ions in combination with time-of-flight mass spectrometry. Anal Chem 84:4095-4103

Brown L J, Toutoungi D E, Devenport N A, Reynolds J C, Kaur-Atwal G, Boyle P, \& Creaser C S (2010) Miniaturized ultra high field asymmetric waveform ion mobility spectrometry combined with mass spectrometry for peptide analysis. Anal Chem 82:9827-9834

Catinella S, Pelizzi N, Marsilio R, Zanol M, Porcelli B, Terzuoli L, Giubbolini M, \& Setacci C (2001) Determination of purine compounds in carotid artery plaque by liquid chromatography electrospray ionization tandem mass spectrometry. J Mass Spectrom 36:441-2

Chan S-H, Hung C-H, Shihd J-Y, Chu P-M, Cheng Y-H, Lin H-C, HsiehP-L, Tsai K-L (2018)

Exercise intervention attenuates hyperhomocysteinemia-induced aortic endothelial oxidative injury by regulating SIRT1 through mitigating NADPH oxidase/LOX-1 signaling. Redox Biol 14:116-125

Chiappin S, Antonelli G, Gatti R, \& Palo E F De (2007) Saliva specimen: A new laboratory tool for diagnostic and basic investigation. Clin Chim Acta 383:30-40

Dame Z T, Aziat F, Mandal R, \& Krishnamurthy R (2015) The human saliva metabolome.

Metabolomics 11:1864-1883

Daskalaki E, Blackburn G, Kalna G, Zhang T, Anthony N, \& Watson D G (2015) A Study of the Effects of Exercise on the Urinary Metabolome Using Normalisation to Individual Metabolic Output. Metabolites 5:119-139

Daskalaki E, Easton C, \& Watson D G (2014) The Application of Metabolomic Profiling to the 
Effects of Physical Activity. Curr Metabolomics 2:233-263

Finsterer J (2012) Biomarkers of peripheral muscle fatigue during exercise. BMC Musculoskelet Disord 13:1-13

Finsterer J, \& Drory V E (2016) Wet, volatile, and dry biomarkers of exercise-induced muscle fatigue. BMC Musculoskelet Disord 17:40

Flanders M M, Crist R A, Roberts W L, \& Rodgers G M (2005) Pediatric reference intervals for seven common coagulation assays. Clin Chem 51:1738-1742

Guan Y, Chu Q, \& Ye J (2004) Determination of uric acid in human saliva by capillary electrophoresis with electrochemical detection: Potential application in fast diagnosis of gout. Anal Bioanal Chem 380:913-917

Guan Y, Wu T, \& Ye J (2005) Determination of uric acid and p-aminohippuric acid in human saliva and urine using capillary electrophoresis with electrochemical detection: Potential application in fast diagnosis of renal disease. J Chromatogr B Analyt Technol Biomed Life Sci 821:229-234

Halliwell B, \& Chirico S (1993) Lipid peroxidation: its mechanism, measurement and significance. Am J Clin Nutr 57: 715S-725S

Hellsten-Westing Y, Kaijser L, Ekblom B, \& Sjödin B (1994) Exchange of purines in human liver and skeletal muscle with short-term exhaustive exercise. Am J Physiol 266:R81-R86

Ito T, van Kuilenburg a B, Bootsma a H, Haasnoot a J, van Cruchten A, Wada Y, \& van Gennip a $\mathrm{H}$ (2000) Rapid screening of high-risk patients for disorders of purine and pyrimidine metabolism using HPLC-electrospray tandem mass spectrometry of liquid urine or urine-soaked filter paper strips. Clin Chem 46:445-452

Ji L L (2015) Redox signaling in skeletal muscle: role of aging and exercise. Adv Physiol Educ $39: 352-359$

Kochanska B, Smolenski R T, \& Knap N (2000) Determination of adenine nucleotides and their metabolites in human saliva. Acta Biochim Pol 47:877-79

Ligtenberg A J M, Brand H S, Van Den Keijbus P A M, \& Veerman E C I (2015) The effect of physical exercise on salivary secretion of MUC5B, amylase and lysozyme. Arch Oral Biol 60:16391644

Lu W, Kimball E, \& Rabinowitz J D (2006) A high-performance liquid chromatography-tandem mass spectrometry method for quantitation of nitrogen-containing intracellular metabolites. J Am Soc Mass Spectrom 17:37-50

Malkar A, Devenport N A, Martin H J, Patel P, Turner M A, Watson P, Maughan R J, Reid H J, Sharp B L, Thomas C L P, Reynolds J C, \& Creaser C S (2013) Metabolic profiling of human saliva before 
and after induced physiological stress by ultra-high performance liquid chromatography-ion mobilitymass spectrometry. Metabolomics 9:1192-1201

Margonis K, Fatouros I G, Jamurtas A Z, Nikolaidis M G, Douroudos I, Chatzinikolaou A, Mitrakou A, Mastorakos G, Papassotiriou I, Taxildaris K, \& Kouretas D (2007) Oxidative stress biomarkers responses to physical overtraining: Implications for diagnosis. Free Radic Biol Med 43:901-910 Markelj J, Zupančić T, \& Pihlar B (2016) Optimization of high performance liquid chromatography method for simultaneous determination of some purine and pyrimidine bases. Acta Chim Slov 63:817

Murray A W (1966) Purine-Phosphoribosyltransferase Activities in Rat and Mouse Tissues and in Ehrlich Ascites-Tumour Cells. Biochem J 100:664-670

Ong E S, Zou L, Li S, Cheah P Y, Eu K W, \& Ong C N (2010) Metabolic profiling in colorectal cancer reveals signature metabolic shifts during tumorigenesis. Mol Cell Proteomics 1-42

Radak Z, Chung H Y, \& Goto S (2008) Systemic adaptation to oxidative challenge induced by regular exercise. Free Radic Biol Med 44:153-159

Smith R W, Toutoungi D E, Reynolds J C, Bristow A W T, Ray A, Sage A, Wilson I D, Weston D J, Boyle B, \& Creaser C S (2013) Enhanced performance in the determination of ibuprofen 1- $\beta$-O-acyl glucuronide in urine by combining high field asymmetric waveform ion mobility spectrometry with liquid chromatography-time-of-flight mass spectrometry. J Chromatogr A 1278:76-81

Soukup M, Biesiada I, Henderson A, Idowu B, Rodeback D, Ridpath L, Bridges E G, Nazar A M, \& Bridges K (2012) Salivary uric acid as a noninvasive biomarker of metabolic syndrome. Diabetol Metab Syndr 4:14

Speranza L, Grilli A, Patruno A, Franceschelli S, Felzani G, Pesce M, Vinciguerra I, de Lutiis M A, \& Felaco M (2007) Plasmatic markers of muscular stress in isokinetic exercise. J Biol Regul Homeost Agents 21:23-31

Valko M, Leibfritz D, Moncol J, Cronin M T, Mazur M, \& Telser J (2007) Free radicals and antioxidants in normal physiological functions and human disease. Int.J Biochem Cell Biol. 39:44-84 Vollaard N B, Shearman J P, \& Cooper C E (2005) Exercise induced oxidative stress. Myths,realities and physiological relevance. Sports Med 35:1045-1062

Walsh N P (1999) The effects of high-intensity intermittent exercise on saliva IgA, total protein and alpha-amylase. J Sports Sci 17:129-134

Wilson L S, Wadley A J, Gyimah B, Reynolds J C, Mastana S S, \& Lindley M R (2017) Investigation into the effects of fish oil supplementation on cycling performance, oxidative stress and inflammation 
in healthy male cyclists: a randomised, placebo controlled, cross-over study. Am J Clin Nutrition, Under Review

Yigla M, Berkovich Y, \& Nagler R M (2007) Oxidative stress indices in COPD-Broncho-alveolar lavage and salivary analysis. Arch Oral Biol 52:36-43

Zielinski J, Kusy K, \& Rychlewski T (2011) Effect of Training Load Structure on Purine Metabolism in Middle-Distance Runners. Med Sci Sports Exerc 43:1798-1807 